\title{
Genome-level analyses of Mycobacterium bovis lineages reveal the role of SNPs and antisense transcription in differential gene expression
}

Paul Golby ${ }^{1 *}$, Javier Nunez ${ }^{1}$, Adam Witney², Jason Hinds², Michael A Quail ${ }^{3}$, Stephen Bentley ${ }^{3}$, Simon Harris ${ }^{3}$, Noel Smith', R Glyn Hewinson ${ }^{1}$ and Stephen V Gordon ${ }^{4}$

\begin{abstract}
Background: Bovine tuberculosis (bTB) is a disease with major implications for animal welfare and productivity, as well as having the potential for zoonotic transmission. In Great Britain (GB) alone, controlling bTB costs in the region of $£ 100$ million annually, with the current control scheme seemingly unable to stop the inexorable spread of infection. One aspect that may be driving the epidemic is evolution of the causative pathogen, Mycobacterium bovis. To understand the underlying genetic changes that may be responsible for this evolution, we performed a comprehensive genome-level analyses of $4 \mathrm{M}$. bovis strains that encompass the main molecular types of the pathogen circulating in GB.

Results: We have used a combination of genome sequencing, transcriptome analyses, and recombinant DNA technology to define genetic differences across the major M. bovis lineages circulating in GB that may give rise to phenotypic differences of practical importance. The genomes of three M. bovis field isolates were sequenced using Illumina sequencing technology and strain specific differences in gene expression were measured during in vitro growth and in ex vivo bovine alveolar macrophages using a whole genome amplicon microarray and a whole genome tiled oligonucleotide microarray. SNP/small base pair insertion and deletions and gene expression data were overlaid onto the genomic sequence of the fully sequenced strain of $M$. bovis 2122/97 to link observed strain specific genomic differences with differences in RNA expression.

Conclusions: We show that while these strains show extensive similarities in their genetic make-up and gene expression profiles, they exhibit distinct expression of a subset of genes. We provide genomic, transcriptomic and functional data to show that synonymous point mutations (sSNPs) on the coding strand can lead to the expression of antisense transcripts on the opposing strand, a finding with implications for how we define a 'silent' nucleotide change. Furthermore, we show that transcriptomic data based solely on amplicon arrays can generate spurious results in terms of gene expression profiles due to hybridisation of antisense transcripts. Overall our data suggest that subtle genetic differences, such as sSNPS, may have important consequences for gene expression and subsequent phenotype.
\end{abstract}

Keywords: Bovine tuberculosis, Mycobacterium bovis, Microarray, Transcript, SNP, Antisense, Macrophage

\footnotetext{
* Correspondence: paul.golby@ahvla.gsi.gov.uk

${ }^{1}$ Animal Health and Veterinary Laboratories Agency, Woodham Lane, New Haw Addlestone, Surrey KT15 3NB, UK

Full list of author information is available at the end of the article
} 


\section{Background}

Mycobacterium bovis is the causative agent of bovine tuberculosis (bTB), an endemic disease of cattle in Great Britain (GB) with the potential for zoonotic transmission to humans. In GB the primary control of bTB is through 'test and slaughter' surveillance, whereby cattle that are positive to the tuberculin skin test [1] are removed from the herd and slaughtered. In spite of this approach, which has been in place since the 1950s, the number of TB-positive cattle slaughtered is increasing year on year - approximately 30,000 cattle were tested and slaughtered between 2012-2013, compared to 300 between 1995-1996 (http://www.defra.gov.uk/animal-diseases/a-z/ bovine-tb/). The UK (GB and Northern Ireland) governments currently spend approximately $£ 100$ million per year collectively on control measures and compensation to farmers for slaughtered cattle. The failure of the testand-slaughter policy to control the spread of infection in large parts of GB suggests that we need a much greater understanding of the TB disease dynamic, including the role of pathogen diversity as a potential driver of this process.

$M$. bovis isolates that are cultured from skin test-reactor animals are currently genetically typed using a combination of spoligotyping [2] and VNTR [3]. Spoligotyping exploits a polymorphic region of the genome called the DR locus which consists of multiple, identical 36bp repeats interspersed with unique sequences known as spacers. Isolates of $M$. bovis differ in the presence or absence of spacers and adjacent DRs, allowing a 'barcode' to be generated for each molecular type. Spoligotypes 9 and 17 are the dominant molecular types in the UK, with more than one third of all isolates corresponding to Type 9 and a quarter to Type 17. VNTR measures the variation at repeat sequences in 6 regions of the genome. There are 6 major VNTR types for Type 9, while all others show only one dominant profile, suggesting that $M$. bovis Type 9 strains are more genetically variable compared with other spoligotypes. Integration of molecular typing with geographical information systems allows temporal and spatial distribution of molecular types to be mapped across GB. Type 9 isolates are widely distributed across GB, while type 17 is an emerging clone which has expanded out of foci around Gloucester, Hereford and Worcester. Similarly, Types 25 and 35 have expanded out of Staffordshire/Shropshire and Hereford/Worcester, respectively. Between them, types 25, 35, 9 and 17 encompass the diversity of the major clonal lineages of $M$. bovis circulating in the UK.

An analysis of molecular typing data from $\sim 11,500$ $M$. bovis isolates revealed that the population structure of $M$. bovis in GB could not be explained by random mutation and drift and instead, it appeared that certain strains were increasing at a faster rate relative to others
[4]. One suggestion for the 'clonal expansion' of GB $M$. bovis genotypes was that certain genotypes had a selective advantage over others leading to an increase in their frequency in the population [4]. Supportive of this hypothesis, several lines of evidence have suggested that M. bovis isolates show phenotypic differences to each other. Fourier-Transform Infrared Spectroscopy (FT-IR) has been used to generate metabolic profiles of the 10 major spoligotype groups of $M$. bovis isolates circulating in GB. Clustering analysis of the resulting spectra showed that the spectra could be differentiated according to spoligotype, indicating that strains of different spoligotypes possess phenotypically distinct traits [5]. In addition, it has also been shown that type 17 isolates have lower incorporation rates of propionate into membrane lipid components compared to other field strains, suggesting a degree of metabolic remodelling in the type 17 lineage [6]. Hence it appears that genetic differences across $M$. bovis lineages may impact on phenotypic traits. This latter finding may have important implications for vaccine and diagnostic test development, in terms of which experimental challenge strains to test vaccines against or on influencing diagnostic test performance.

In an attempt to better define genetic differences across the major $M$. bovis lineages circulating in GB that may give rise to phenotypic differences of practical importance, we have used a combination of genome sequencing, transcriptome analyses, and recombinant DNA technology. The genomes of three M. bovis field isolates were sequenced using Illumina sequencing technology and strain specific differences in gene expression were measured during in vitro growth and in ex vivo bovine alveolar macrophages $(\mathrm{M} \phi)$ using a whole genome amplicon microarray. Recent discoveries of small non coding RNA within mycobacteria $[7,8]$ prompted us to assess differences in sRNA expression across the isolates using a whole genome tiled oligonucleotide microarray. SNP/small base pair insertion and deletions (INDELs) and gene expression data were overlaid onto the genomic sequence of the fully sequenced strain of M. bovis 2122/97 to link observed strain specific genomic differences with differences in RNA expression.

\section{Results}

Comparative genomics of $M$. bovis field isolates using whole genome sequencing and microarrays

The strains for this study were chosen to reflect the genomic diversity of the $M$. bovis population circulating in GB, and are listed in Table 1. M. bovis strains were typed using a combination of spoligotyping and VNTR. For each spoligotype group, an isolate which possessed the most common VNTR profile was selected, so that 
Table $1 M$. bovis field strains used in this study

\begin{tabular}{lccccc}
\hline AHVLA ID & International spoligotype ID [9] & UK spoligotype ID & VNTR ID & Year of isolation & UK county of isolation \\
\hline $1121 / 01$ & SB0263 & 17 & $7555^{*} 33.1$ & 2001 & Wiltshire \\
$2122 / 97$ & SB0140 & 9 & $8555^{*} 33.1$ & 1997 & Devon \\
$2451 / 01$ & SB0129 & 25 & $6554^{*} 23.1$ & 2001 & Clwyd \\
$1307 / 01$ & SB0134 & 35 & $3354^{*} 33.1$ & 2001 & Shropshire \\
\hline
\end{tabular}

${ }$ indicates partial number of repeats.

each chosen strain was the most representative of each spoligotype group (Table 1). Of the four studied strains, 2451/01 and 1307/01 diverged earliest during descent from the most recent common ancestor of $M$. bovis in GB and are more distant to strains 1121/01 and 1307/ 01 (Smith, N. personal communication). All 4 strains were isolated from diseased cows belonging to herds which were taken from farms in geographically diverse areas of the country.

The genomes of the three $M$. bovis strains 1121/01, 2451/01 and 1307/01 were paired-end sequenced using Illumina sequencing technology. Processed sequence reads were mapped to the genome of the fully sequenced and annotated strain 2122/97 [10] to identify SNPs. SNPs were identified across all four sequenced $M$. bovis strains, and their positions, together with their SNP class, are listed in Additional file 1. The evolutionary relationships between the three sequenced strains are depicted in Figure 1 using a phylogenetic tree and a distance matrix plot. Three genome sequenced members of the Mycobacterium tuberculosis complex, M. bovis BCG Pasteur, M. tuberculosis H37Rv and M. africanum GM041182, were included in the analysis to place the $M$. bovis strains in a wider mycobacterial context. The numbers of SNPs between M. bovis 2122/97 and the three newly sequenced $M$. bovis strains were found
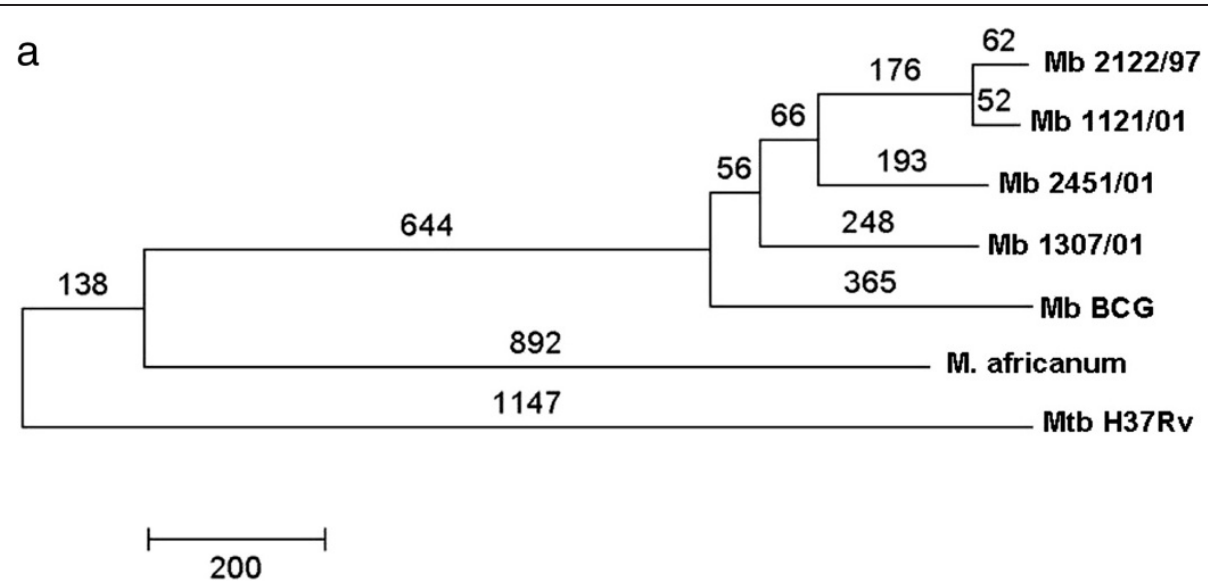

b

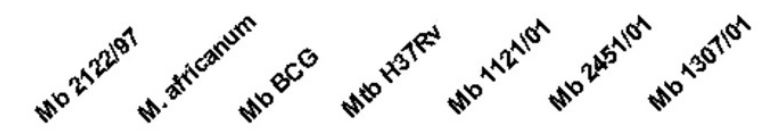

\begin{tabular}{|c|c|c|c|c|c|c|c|}
\cline { 2 - 8 } Mb 2122/97 & 0 & 1887 & 717 & 2280 & 114 & 431 & 552 \\
\cline { 2 - 8 } M. africanum & 1887 & 0 & 1897 & 2177 & 1878 & 1847 & 1834 \\
\cline { 2 - 8 } Mb BCG & 717 & 1897 & 0 & 2290 & 710 & 679 & 668 \\
\cline { 2 - 8 } Mtb H37Rv & 2280 & 2177 & 2290 & 0 & 2273 & 2240 & 2227 \\
\hline Mb 1121/01 & 114 & 1878 & 710 & 2273 & 0 & 421 & 542 \\
\hline Mb 2451/01 & 431 & 1847 & 679 & 2240 & 421 & 0 & 505 \\
\hline Mb 1307/01 & 552 & 1834 & 668 & 2227 & 542 & 505 & 0 \\
\hline
\end{tabular}

Figure 1 Whole genome SNP-based evolutionary analysis of $\boldsymbol{M}$. bovis sequenced strains. (a). Phylogenetic tree with numbers above the branches indicating the number of SNPs identified between the organism and its common ancestor. (b) Distance matrix plot showing the number of SNPS present between selected pairs of strains. 
to be consistent with their predicted evolutionary distances from each other. Strain 1121/01 (type 17) is most closely related to the original genome sequenced strain 2122/97 (type 9) with 114 SNPs, whereas the more distantly related strains 2451/01 (type 25) and 1307/01 (type 35) have 431 and 552 SNP differences respectively. All four sequenced $M$. bovis strains were more distantly related to $M$. africanum ( 1800 SNPs) and M. tuberculosis H37Rv ( 2200 SNPs).

In addition to SNPs, both large and small INDELs can be inferred from NGS data, although there are several challenges involved [11]. Large sized insertions $(>500$ bp) are particularly difficult to identify with accuracy as they require the de novo assembly of the reads that fail to map to the reference genome and the subsequent database searching with the resulting contigs. Accurate identification of large sized deletions (>500 bp) are, however, more easily identifiable and Additional file 2 lists those that have been identified by NGS in the genomes of the three sequenced strains. As poor coverage can confound the identification of deletions, we sought to use microarray technology to confirm the deletions identified by the NGS data. Genomic DNA was isolated from all four strains, labelled with fluorescent dyes and hybridised to a whole genome $M$. tuberculosis $/ M$. bovis amplicon microarray (see Methods). Table 2 lists several LSPs that were detected across the strains using microarrays. Both NGS and microarray data predict the presence of the large $6.8 \mathrm{~kb}$ deletion (RDbovis(d)_0173) which encompasses genes Mb1963-Mb1971 and appears to be specific to UK strains belonging to Type 17 that has been described in a previous study [12]. Several of these gene products are predicted to encode proteins involved in lipid metabolism, but the lipid composition of several type 17 isolates was found to be no different to other $M$. bovis strains, although their ability to incorporate propionate into mycolic acids was found to be lower [6]. A smaller $1.6 \mathrm{~kb}$ deletion specific 1307/01 that comprises the 3' end of Mb2056c, Mb2055c and the 5' end of $p k f B(\mathrm{Mb} 2054 \mathrm{c})$ was also detected by both NGS and microarray data, Due to a single base deletion, Mb2056c and Mb2055c are pseudogenes in 2122/97, but the two genes exist as one intact functional gene in 2145 and H37Rv (Rv2030c). The pfkB gene encodes a phosphofructokinase homologue and is strongly immunogenic in human TB patients, while Rv2030c encodes an erythromycin esterase. Both $p f k B$ and Mb2056c are members of the DosR regulon, which are highly upregulated under anaerobic conditions and have been implicated in bacterial persistence in vivo [13]. Other smaller deletions detected include a deletion of a probable lipid transfer protein encoding gene Mb1699c, which is specific to $1307 / 01$, and an aldo/keto reductase encoding gene, Mb2320 that is specific to $1121 / 01$.

\section{Linking SNPs to genes that show differential expression amongst $M$ bovis strains grown under vitro conditions and in ex vivo macrophages}

The four M. bovis field strains were grown to midlogarithmic phase in pyruvate-containing Middlebrook 7H9 liquid media, and then used to infect bovine alveolar M $\phi$ using a multiplicity of infection (MOI) of 10:1 (bacilli: $M \phi$ ). Mycobacterial RNA was recovered from infected host cells 4 and 24 hrs post infection using a differential lysis procedure and amplified using a modified procedure similar to that described by van Gelder et al. ([14]; see Methods). As a control, RNAs were also extracted from strains that had been incubated statically in RPMI cell culture media for a period of $4 \mathrm{hrs}$. To eliminate potential skewing effects on the transcriptome resulting from the amplification process, comparisons were made only between amplified RNA generated from samples collected at the same time point and biological replicate. For the in vitro growth condition, total RNAs were extracted from the four strains grown in a pyruvatecontaining Middlebrook 7H9 liquid media and rolled during incubation.

Table 2 Large sequence polymorphisms present in $M$. bovis field isolates

\begin{tabular}{|c|c|c|c|c|c|c|c|c|c|}
\hline $\mathrm{Mb}$ CDS & Mtb CDS & $\begin{array}{l}\text { Genomic postn. } \\
\text { (wrt to } 2122 / 97 \text { ) }\end{array}$ & $\begin{array}{c}\text { Size of } \\
\text { deletion (bp) }\end{array}$ & Gene(s) & $2122 / 97$ & $1121 / 01$ & $2451 / 01$ & $1307 / 01$ & Product(s) \\
\hline Mb1699c & Rv1627c & $n / d$ & $n / d$ & & & & & DEL & Probable lipid transfer protein \\
\hline $\begin{array}{l}\text { aMb1963c- } \\
\text { Mb1971 }\end{array}$ & $\begin{array}{l}\text { Rv1928c- } \\
\text { Rv1936 }\end{array}$ & $2172231-2179038$ & 6807 & $\begin{array}{l}t p x, \text { fadE17, } \\
\text { fadE18, echA13 }\end{array}$ & & DEL & & & $\begin{array}{l}\text { Gene products have roles in } \\
\text { lipid metabolism }\end{array}$ \\
\hline Mb2054c & Rv2029c & $2259798-2261457$ & 1659 & $p f k B$ & & & & DEL & Possible phosphofructokinase \\
\hline $\begin{array}{l}\text { Mb2055c/ } \\
\text { Mb2056c }\end{array}$ & Rv2030c & & & & & & & DEL & $\begin{array}{l}\text { Conserved hypothetical protein } \\
\text { (frame-shifted in 2212/97) }\end{array}$ \\
\hline Mb2320 & Rv2298 & $n / d$ & $n / d$ & & DEL & DEL & & & Aldo/keto reductase \\
\hline Mb3923c & Rv3894c & $n / d$ & $n / d$ & & & & DEL & & $\begin{array}{l}\text { Conserved membrane protein } \\
\text { (frame-shifted in } 2212 / 97 \text { ) }\end{array}$ \\
\hline
\end{tabular}


The RNAs extracted from each of the growth conditions were converted to cyanine labelled cDNA using reverse transcriptase and hybridised to whole genome amplicon microarrays. Using only those genes that are common to all four strains, we found a total set of 70 genes that showed a 2.5 -fold or more difference in expression in one or more strains when pairwise comparisons were made between the transcriptomes of 2122/97 and $1121 / 01,2451 / 01$ or $1307 / 01$ (Additional file 3). A subset of these 70 genes is shown in Table 3 where key examples of alterations in metabolic processes are shown. The numbers of genes that were found to show differential expression across the four strains reflected the evolutionary distances between 2122/97 and the other 3 strains. Thus, 1121/01, which is closely related to $2122 / 97$, shows only 5 differentially expressed genes, while the most distantly related strain 1307/01 shows 56 genes differently expressed compared to 2122/97. Of these 56 genes, 5 were specific to the in vitro condition, while 19 were specific to the M $\phi$. Ten genes were common to both conditions, which serves to validate the technical reproducibility of the RNA amplification process.

Using the genome sequencing information determined for each of the four strains, we attempted to correlate the observed strain-specific differences in gene expression with the presence or absence of mutations within the coding regions or promoters of those genes that show differential gene expression, or in genes that are known to regulate the activity of those genes. Mb1749c and Mb1750c are two genes that are specifically upregulated in 1307/01 and encode a toxin and antitoxin (TA) pair, respectively, belonging to type II TA systems of the VapBC family [15]. Members of VapB type toxins contain PIN domains that cleave RNA and thus function to control translation of mRNA transcripts [16]. The homologous genes from strain 1307/01 show up to 19and 10-fold higher levels of expression, respectively, than those of the other three strains. An analysis of the coding sequences of Mb1749c across all four strains revealed that the 1307/01 homologue has a unique nSNP at position 1932704 (wrt 2122/97 genomic sequence), a $\mathrm{C}$ - $\mathrm{T}$ transition that results in the nonconservative substitution of Gly19 to Asp. Research has shown that TA gene pairs negatively regulate their own expression through binding of the TA protein complex to the promoter region of the TA gene pair, thus preventing access to RNA polymerase [17]. The G19D mutation in Mb1749c could therefore impair the ability of the complex to bind to the promoter resulting in the deregulation of the TA gene pair.

Mb2007c, which shows a 4-fold higher expression in $1307 / 01$ only, encodes a transcriptional regulator of the LysR class. There are two SNPs present in the coding sequence of Mb2007c in 1307/01 which are absent in the homologues of the other three strains: the first is a nSNP which results in the conservative substitution of Arg137 to Gln, while the second is a more debilitating nonsense SNP, which ultimately leads to a protein whose length is only $60 \%$ that of the wild-type. Many regulators belonging to the LysR family regulate their own expression through a negative autoregulatory mechanism similar to that described above for VapBC TA systems [18]. A loss in protein integrity could, therefore, result in the regulator being unable to bind the regulatory region, leading to the observed upregulation in the expression of this gene in $1307 / 01$. As the product of this gene is predicted to be a transcriptional regulator, it was speculated that the regulation of gene(s) controlled by regulator could be affected in 1307/01 due to the severely truncated form of this protein. As LysR regulators are often found to regulate genes that are divergently transcribed from the lysR gene, it was surprisingly to find that expression of the Mb2008 homologue in 1307/01, which is predicted to encode a lysine transporter, does not show any difference in expression in 1307/01 to $2122 / 97$. To define the regulon of this regulator, we first compared the transcriptomes of 2122/97 transformed with a multicopy plasmid expressing the truncated copy of $m b 2007 c$ against a vector only control. No differences in expression were found (data not shown), which could indicate that the regulator does not control any other genes apart from itself, or that experimental conditions did not favour the active form of the regulator. LysR regulators regulate expression of their regulon through binding of a co-inducer to the C-terminal domain, and the failure to observe any changes in gene expression could therefore be due to the absence of the co-inducer during the experiment. A further experiment to compare the profiles of $2122 / 97$ expressing either the truncated or wild type forms of the protein also showed no differences in expression (data not shown).

Nitrite reductase catalyses the reduction of nitrite to ammonia and is strongly expressed during growth in the presence of nitrate or nitrite, but repressed in the presence of ammonia [19]. The gene encoding the large subunit of the nitrite reductase, nirB (Mb0258), shows approximately 9-fold higher expression in 1307/01 compared to the other 3 strains in our standard ammonia containing 7H9 growth media, suggesting that the strain has lost regulatory control of this gene. Expression of nirB in $M$. tuberculosis has been shown to be controlled by the response regulator $G \ln R$ [20], but an analysis of the sequence of the $g \ln R$ orthologue from all four strains revealed no differences in either the coding or promoter sequences. A comparison of the nirB sequence from all 4 strains did, however, reveal the presence of a single base $(\mathrm{C}$ to $\mathrm{T})$ transition leading to a sSNP that is specific 
Table 3 Fold change differences in gene expression in M. bovis field isolates 1121, 2451 and 1307 compared to 2122

\begin{tabular}{|c|c|c|c|c|c|c|c|c|c|c|c|c|c|c|c|c|}
\hline $\mathrm{Mb}$ CDS & Mtb CDS & Common & & & $1121 / 01$ & & & & $2451 / 01$ & & & & $1307 / 01$ & & Product & Assoc. SNP/InDel \\
\hline & & & 7h9 & RPMI & $4 \mathrm{hr} M \varnothing$ & $24 \mathrm{hr} M \varnothing$ & 7h9 & RPMI & $4 \mathrm{hr} M \varnothing$ & $24 \mathrm{hr} M \varnothing$ & 7h9 & RPMI & $4 \mathrm{hr} M \varnothing$ & $24 \mathrm{hr} M \varnothing$ & & \\
\hline Mb0038c & Rv0037c & & & & & & & $4 \uparrow$ & $3 \uparrow$ & $3 \uparrow$ & & & & & $\begin{array}{l}\text { probable conserved } \\
\text { integral membrane } \\
\text { protein }\end{array}$ & \\
\hline $\mathrm{Mb0124c}$ & Rv0120c & fusA2 & & & & & $3 \uparrow$ & & & & $3 \uparrow$ & & & & $\begin{array}{l}\text { probable elongation } \\
\text { factor }\end{array}$ & \\
\hline Mb0258 & Rv0252 & $\operatorname{nir} B$ & & & & & & & & & $9 \uparrow$ & $12 \uparrow$ & $21 \uparrow$ & $9 \uparrow$ & $\begin{array}{l}\text { nitrite reductase } \\
\text { (large subunit) }\end{array}$ & $\begin{array}{c}\text { sSNP at postn. } 303227 \text { in 1307/01 } \\
\text { only; C-T; }\end{array}$ \\
\hline Mb0259 & Rv0253 & nirD & & & & & & & & & & $3 \uparrow$ & $4 \uparrow$ & $5 \uparrow$ & $\begin{array}{l}\text { nitrite reductase } \\
\text { (small subunit) }\end{array}$ & \\
\hline $\mathrm{Mb0428c}$ & Rv0420c & & & & & & & & & & & & & $2 \uparrow$ & $\begin{array}{l}\text { possible } \\
\text { transmembrane } \\
\text { protein }\end{array}$ & \\
\hline Mb0734 & Rv0713 & & & & & & & & $2 \uparrow$ & & & & & & $\begin{array}{l}\text { probable conserved } \\
\text { transmembrane } \\
\text { protein }\end{array}$ & \\
\hline Mb0947c & Rv0923c & & & & & & $2 \downarrow$ & & & & $2 \downarrow$ & & & & $\begin{array}{c}\text { conserved } \\
\text { hypotheitical protein }\end{array}$ & \\
\hline Mb0948c & Rv0924c & mntH & & & & & & & & & & & & & cation uptake system & \\
\hline $\begin{array}{l}\text { Mb1013; } \\
\text { Mb1014 }\end{array}$ & Rv0987 & & & & & & $5 \uparrow$ & $10 \uparrow$ & $5 \uparrow$ & & $5 \uparrow$ & $7 \uparrow$ & $6 \uparrow$ & & $\begin{array}{l}\text { probable adhesion } \\
\text { component transport } \\
\text { ABC transporter }\end{array}$ & $\begin{array}{l}\text { nSNP at postn. } 1104263 \text { in } 2451 / 01 \text { and } \\
1307 / 01 ; A-G \text { (STOP to W); SSNP at } \\
\text { postn } 1103991 \text { in } 2451 / 01 \text { and } 1307 / 01\end{array}$ \\
\hline Mb1015 & Rv0988 & & & & & & $3 \uparrow$ & $9 \uparrow$ & & & $3 \uparrow$ & $5 \uparrow$ & $5 \uparrow$ & & $\begin{array}{l}\text { possible conserved } \\
\text { exported protein }\end{array}$ & \\
\hline Mb1161 & Rv1130 & & & & & & & & & & & & & $2 \downarrow$ & $\begin{array}{l}\text { conserved } \\
\text { hypothetical protein }\end{array}$ & \\
\hline Mb1162 & Rv1131 & $g \mid t A 1$ & & & & & $3 \downarrow$ & & & & & & & & $\begin{array}{l}\text { probable citrate } \\
\text { synthase }\end{array}$ & \\
\hline Mb1554c & Rv1527c & pks5 & & & $2 \downarrow$ & & & & & & & & & & $\begin{array}{l}\text { probable polyketide } \\
\text { synthase }\end{array}$ & \\
\hline Mb1562 & Rv1535 & & & $2 \uparrow$ & & & & & & & & $5 \uparrow$ & & & hypothetical protein & \\
\hline Mb1619c & Rv1593c & & & & & $2 \uparrow$ & & & & & & & & & $\begin{array}{c}\text { conserved } \\
\text { hypothetical protein }\end{array}$ & \\
\hline Mb1749c & Rv1720c & & & & & & & & & & $10 \uparrow$ & $19 \uparrow$ & $18 \uparrow$ & $12 \uparrow$ & $\begin{array}{c}\text { conserved } \\
\text { hypothetical protein }\end{array}$ & $\begin{array}{l}\text { nSNP at postn. } 1932704 \text { in } \\
1307 / 01 \text { only ; C-T (G to D) }\end{array}$ \\
\hline Mb1750c & Rv1721c & & & & & & & & & & $6 \uparrow$ & $3 \uparrow$ & $9 \uparrow$ & $5 \uparrow$ & $\begin{array}{c}\text { conserved } \\
\text { hypothetical protein }\end{array}$ & \\
\hline Mb1833c & Rv1804 & & & & & & & & & & & & & $3 \downarrow$ & $\begin{array}{c}\text { conserved } \\
\text { hypothetical protein }\end{array}$ & \\
\hline Mb1834c & Rv1805c & & & & & & & & & & & $4 \uparrow$ & & & hypothetical protein & \\
\hline
\end{tabular}


Table 3 Fold change differences in gene expression in M. bovis field isolates 1121, 2451 and 1307 compared to 2122 (Continued)

\begin{tabular}{|c|c|c|c|c|c|c|c|c|c|c|c|c|c|}
\hline Mb1835 & Rv1806 & PE20 & & & & & & & $3 \uparrow$ & & & PE family protein & \\
\hline Mb1885c & Rv1854c & ndh & & & & & & & & & $3 \downarrow$ & $\begin{array}{l}\text { probable nadh } \\
\text { dehydrogenase }\end{array}$ & \\
\hline Mb1914c & Rv1882C & & & & $6 \uparrow$ & $3 \uparrow$ & $6 \uparrow$ & & & & & $\begin{array}{c}\text { probable short-chain } \\
\text { type dehydrogenase/ } \\
\text { reductase }\end{array}$ & $\begin{array}{l}\text { sSNP at postn } 2122970 \text { in } \\
2415 / 01 \text { only: C-T }\end{array}$ \\
\hline Mb2007c & Rv1985c & & & & & & & $4 \uparrow$ & $3 \uparrow$ & $3 \uparrow$ & & $\begin{array}{l}\text { probable } \\
\text { transcriptional } \\
\text { regulatory protein }\end{array}$ & $\begin{array}{l}\text { nSNP at postn. } 2208296 \text { in 1307/01 } \\
\text { only; G-A (Q to Stop) }\end{array}$ \\
\hline Mb2015c & RV1992C & $\operatorname{ctp} G$ & $2 \downarrow$ & & & & & & & & $3 \downarrow$ & $\begin{array}{l}\text { probable cation } \\
\text { transporter }\end{array}$ & \\
\hline Mb2420c & Rv2398c & cysW & & & $2 \downarrow$ & & & & $6 \downarrow$ & & & sulphate transporter & \\
\hline Mb2421c & Rv2399c & cyst & & & $2 \downarrow$ & & & & $4 \downarrow$ & & & sulphate transporter & \\
\hline Mb2607 & Rv2577 & & & $9 \downarrow$ & $5 \downarrow$ & $7 \downarrow$ & $12 \downarrow$ & $10 \downarrow$ & $7 \downarrow$ & $7 \downarrow$ & $13 \downarrow$ & $\begin{array}{l}\text { conserved } \\
\text { hypothetical protein } \\
\text { [first part] }\end{array}$ & \\
\hline Mb3194 & Rv3169 & & & & & & & $3 \uparrow$ & $3 \uparrow$ & $2 \uparrow$ & $3 \uparrow$ & $\begin{array}{l}\text { conserved } \\
\text { hypothetical protein }\end{array}$ & \\
\hline Mb3477c & Rv3447c & & & $8 \downarrow$ & & $3 \downarrow$ & $10 \downarrow$ & $9 \downarrow$ & $2 \downarrow$ & & $7 \downarrow$ & $\begin{array}{l}\text { probable conserved } \\
\text { membrane protein }\end{array}$ & $\begin{array}{l}\text { nSNP at postn. } 3812465 \text { in } 2451 / 01 \\
\text { and } 1307 / 01 ; \mathrm{T}-\mathrm{C} \text { (S to G) }\end{array}$ \\
\hline Mb3563c & Rv3533c & PPE62 & & $5 \uparrow$ & $5 \uparrow$ & & & & & & & ppe family protein & \\
\hline Mb3721c & Rv3696c & glpK & & & & & & & & $3 \downarrow$ & & glycerol kinase & \\
\hline Mb3803 & Rv3774 & echA21 & & & $8 \uparrow$ & $7 \uparrow$ & $22 \uparrow$ & & & & & $\begin{array}{l}\text { possible enoyl-coA } \\
\text { hydratase }\end{array}$ & $\begin{array}{l}\text { sSNP at postn. } 4155803 \text { in } \\
2415 / 01 \text { only; G-A }\end{array}$ \\
\hline
\end{tabular}

Up and down arrows indicate fold up- and downregulation, respectively, and empty cells indicate no change in expression. 
to $1307 / 01$. It was not readily apparent why a sSNP in the coding sequence of a gene should lead to an increase in expression of that gene, but there are several reports that show sSNPs leading to changes in stability of mRNA transcripts [21,22]. Rv0987 and Rv0988 of M. tuberculosis $\mathrm{H} 37 \mathrm{Rv}$ encode part of an $\mathrm{ABC}$ transporter and a putative secreted hydrolase, respectively. In 2122/97, a single base transition (G-A) introduces a stop codon that splits Rv0987 into the two pseudogenes, Mb1013 and Mb1014. Previous microarray based gene expression studies by our group have shown that Rv0987 and Rv0988 in M. tuberculosis show higher levels of expression than the orthologous Mb1013/Mb1014 and Mb1015, respectively, in M. bovis 2122/97 [23], and in the present study the Mb1013/ Mb1014 and Mb1015 homologues in 2451/01 and 1307/ 01 also showed higher expression (up to 10-fold) than the homologues in 2122/97 and 1121/01. Comparing the sequences of Mb1013/Mb1014 and Mb1015 across all 4 strains indicated that strains that show high expression have the ' $G$ ' allele.

Mb3477c encodes an ATP binding membrane protein, part of the Esx4 secretion system [24], and gene shows up to 10-fold higher expression in 2451/01 and 1307/01 compared to 2122/97. The gene also contains an A to C transition at position 3812465 , a nSNP at position resulting in the non-conservative substitution of a serine to a glycine residue.
Of the 19 genes that show specific differential expression in the $M \phi$, the most notable are Mb1914c and echA21, which show upregulation in $2451 / 01$ only (up to 6- and 23-fold, respectively). Both genes encode proteins that could be involved in lipid metabolism, and both genes contain single sSNPs that are present in 2451/01, but absent in the other three strains.

Real time RT-PCR was used to verify a selection of genes that showed differential gene expression as predicted by the microarray analysis. Figure 2 compares the fold changes in the expression levels of 4 genes as measured by microarray and real time RT-PCR. The nirB and Mb1749c genes were selected because they showed strong upregulation in 1307/01 in both in vitro and ex vivo $\mathrm{M} \phi$ while Mb1914c and echA21 were chosen because the array data predicted them to be specifically upregulated in 2451/01 and only in ex vivo Mф. For each of the 4 genes, the strain dependent pattern of expression as measured by real time RT-PCR was consistent with that measured by microarray, although the fold changes measured by real time RT-PCR were higher than those measured by microarray.

\section{Functional analysis of SNP role in differential gene expression}

The above data showed that many of the strain specific differences in gene expression were linked to the presence
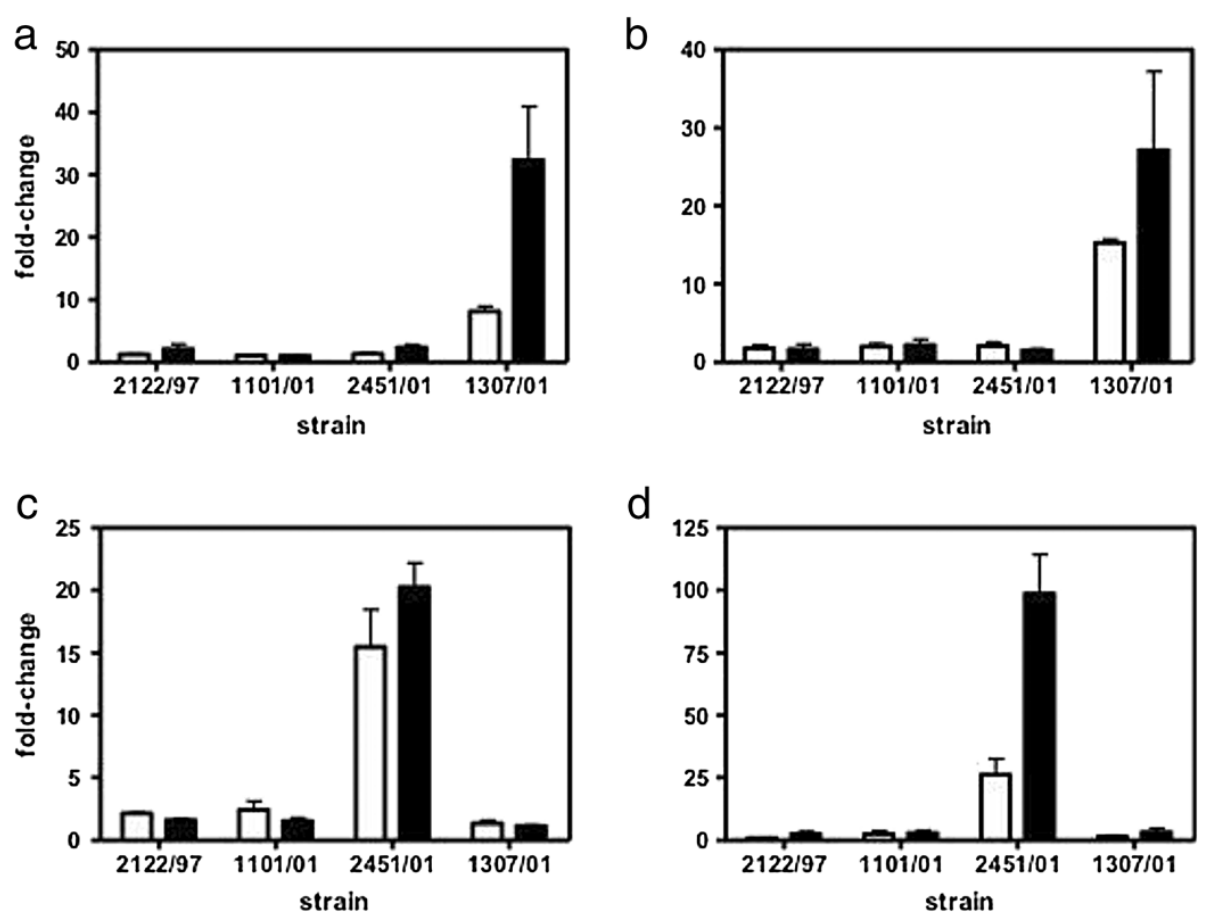

d

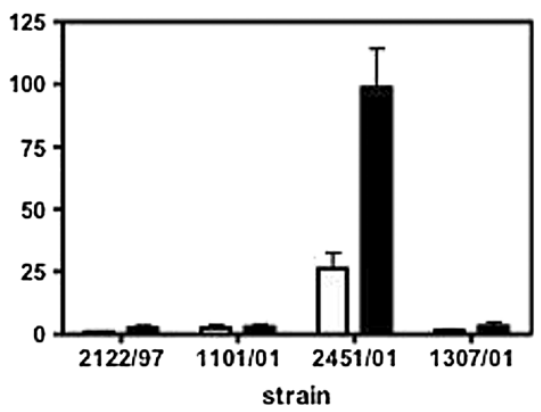

Figure 2 Confirmation of amplicon microarray results with real time RT-PCR. The fold changes in gene expression for (a) Mb1750c, (b) nirB, (c) echA21 and (d) Mb1914C measured by microarray (open bars) in each of the four strains were compared to that measured by real time RT-PCR (closed bars). 
of synonymous or non-synonymous SNPs located within the coding regions of the genes that show variable expression. Non-synonymous SNPs lead to changes in amino acid sequence which can lead to changes in protein function. The $\mathrm{C}$ to $\mathrm{T}$ transition at position 1932704 (wrt 2122/ 97) in the coding sequence of the $1307 / 01 \mathrm{Mb} 1749 \mathrm{c}$ homologue leads to the non-conservative substitution of Gly19 to Asp, and this nSNP appears to be linked to the upregulation of both Mb1749c and Mb1750c in that strain. In order to confirm this, a $0.9 \mathrm{~kb}$ DNA fragment containing the $M b 1749 c-M b 1750 c-M B 1751 c$ region of $1307 / 01$ (containing the ' $\mathrm{T}$ ' allele) and the equivalent region from 2122/97 (with the 'C' allele) were PCR amplified and the fragments were cloned separately into the mycobacterial shuttle vector pKINT (see Methods) to create the constructs pPG107 and pPG106, respectively. The two constructs were introduced into Mycobacterium smegmatis $\mathrm{mc}^{2} 155$, separately, and then the expression of Mb1749c and Mb1750c in M. smegmatis pPG101 was compared to that of $M$. smegmatis pPG102 using real time RT-PCR. Table 4 shows that the expression levels of $\mathrm{Mb} 1750 \mathrm{c}$ and $\mathrm{Mb1749c}$ in the strain expressing the mutated forms of Mb1749c/Mb1750c are 13- and 9-fold higher, respectively, compared to the strain expressing the wild type forms, confirming that this SNP is responsible for the observed up-regulation of the two genes in 1307/01.

Synonymous substitutions do not lead to changes in protein sequence and have generally been considered to be 'silent' or benign. Recent studies, however, have suggested that sSNPs can have functional effects, such as decreased mRNA stability and translation [21,22]. In our own studies, we have found several genes whose expression levels correlate with the presence of sSNPs in the coding regions of those genes. For example, a C-T transversion at position 303227 (wrt 2122/97) within the coding sequence of nirB of $1307 / 01$ is a sSNP that appears to be linked with the upregulation in expression of nirB within that strain. To confirm that this is the case, we PCR amplified $3.5 \mathrm{~kb}$ DNA fragments containing

Table 4 Confirmation of SNP linkage to upregulation in gene expression

\begin{tabular}{lcc}
\hline Strain & Gene & Fold change \\
\hline M. smegmatis $\mathrm{mc}^{2} 155$ (pPG101) & Mb1750c & $16.1 \pm 3.2$ \\
M. smegmatis $\mathrm{mc}^{2} 155$ (pPG101) & Mb1749c & $12.5 \pm 1.2$ \\
M. smegmatis mc ${ }^{2} 155(\mathrm{pPG} 102)$ & Mb1750c & $1.9 \pm 0.8$ \\
M. smegmatis mc ${ }^{2} 155(\mathrm{pPG} 102)$ & Mb1749c & $1.4 \pm 0.4$ \\
Mb 2122/97 (pPG108) & nirB & $2.0 \pm 1.0$ \\
Mb 2122/97 (pPG108) & nirD & $1.3 \pm 0.5$ \\
Mb 2122/97 (pPG109) & nirB & $52.9 \pm 15.9$ \\
Mb 2122/97 (pPG109) & nirD & $3.1 \pm 0.8$ \\
\hline
\end{tabular}

*Fold changes are the mean ratios \pm standard deviation. the hsp-nirB-nirD-cobU region of strain 1307/01 (containing the ' $C$ ' allele) and the equivalent region from strain 2122/97 (with the ' $\mathrm{T}$ ' allele) and cloned them separately into the integrating vector pKINT to create the constructs pPG108 and pPG109, respectively. These constructs were introduced into 2122/97 and the expression levels of nirB and nirD were found to be 30- and 2-fold, respectively, higher in the strain expressing the mutated nirBD locus compared to the strain expressing the wildtype form (Table 4). This confirms that this mutation is responsible for the upregulation of the two genes in this strain.

\section{Use of a high density tiled oligonucleotide microarray to detect differentially expressed small RNA transcripts in M. bovis isolates}

The M. tuberculosis/M. bovis amplicon arrays used in the present study were specifically designed to measure expression levels of genes annotated in the genomic sequence of $M$. bovis 2122/97 [10]. They were not, however, designed to monitor the expression of non-coding RNA such as small RNA within intergenic regions or antisense sRNA. Hence, a high density tiled oligonucleotide microarray consisting of approximately 180,000 partiallyoverlapping (10-base overlap) short 60 mer oligonucleotides was designed that offered an unbiased approach to the detection of strand specific transcripts encoded over the entire $M$. bovis 2122/97 chromosome. Total RNA that includes small sized $(<100 \mathrm{nt})$ RNA species was extracted from the four M. bovis strains that had been grown in liquid media and hybridised to the oligonucleotide microarray. To avoid potential secondary strand synthesis during cDNA synthesis, which could be interpreted as sRNA, the RNA was directly labelled with cyanine based dyes. After pairwise comparisons were performed between 2122/97 and 1121/01, 2451/01 or $1307 / 01,220$ oligonucleotide probes were identified that detected differentially expressed transcripts (2.5 fold cut off) in one or more of the three strains (Additional file 4). Only transcripts detected by multiple ( 2 or more) overlapping probes were regarded as genuine transcripts as those detected by single probes could be due to crosshybridisation effects or represent spurious transcripts. Using these criteria, 26 transcripts, designated T1-T26, were found to show differential expression in one or more of the strains (Table 5), and those transcripts can be divided into those that are encoded within intergenic regions and those encoded within the genomic co-ordinates encompassing annotated coding sequences. Comparison of the differentially expressed gene lists identified using amplicon vs. oligonucleotide arrays (Tables 3 and 5), it is clear that many of the transcripts detected using the amplicon arrays are not necessarily encoded on the sense gene strand, as had been previous interpreted. For 


\begin{tabular}{|c|c|c|c|c|c|c|c|c|c|c|}
\hline Transcript & No. Probes & Position & Size & cds & Strand* & $1121 / 01$ & $2451 / 01$ & $1307 / 01$ & CDS Product & SNP \\
\hline $\mathrm{T} 1$ & 3 & 303138-303295 & 157 & Mb0258/nirB & A & & & $3 \uparrow$ & nitrite reductase (large sub-unit) & \\
\hline $\mathrm{T} 2$ & 5 & $303260-303515$ & 255 & Mb0258/nirB & $\mathrm{s}$ & & & $5 \uparrow$ & nitrite reductase (large sub-unit) & sSNP at 303227 ; C-T in $1307 / 01$ only \\
\hline $\mathrm{T} 3$ & 2 & 1105085-1105193 & 108 & Mb1014 & A & & $3 \uparrow$ & $3 \uparrow$ & $\begin{array}{l}\text { probable adhesion component } \\
\text { of } A B C \text { transporter }\end{array}$ & \\
\hline $\mathrm{T} 4$ & 2 & 1105158-1105315 & 157 & Mb1014 & A & & $3 \uparrow$ & $3 \uparrow$ & $\begin{array}{l}\text { probable adhesion } \\
\text { component of } A B C \text { transporter }\end{array}$ & \\
\hline T5 & 3 & 1105158-1105315 & 157 & Mb1014 & S & & $3 \uparrow$ & $3 \uparrow$ & $\begin{array}{c}\text { probable adhesion } \\
\text { component of } A B C \text { transporter }\end{array}$ & \\
\hline T6 & 5 & $1778876-1779131$ & 255 & Mb1618c & A & $9 \uparrow$ & & & possible secreted lipase & sSNP at $1778879 ;$ C-T in $1121 / 01$ only \\
\hline $\mathrm{T7}$ & 2 & $1840174-1840282$ & 108 & Mb1672c & S & & & $4 \downarrow$ & hypothetical protein & \\
\hline T8 & 3 & 1932158-1932315 & 157 & tRNA-Pro & $\mathrm{S}$ & & & $3 \uparrow$ & proline tRNA & \\
\hline T9 & 5 & 1932452-1932707 & 255 & Mb1749c & S & & & $4 \uparrow$ & $\begin{array}{l}\text { toxin component of } \\
\text { toxin-antitoxin system }\end{array}$ & nSNP at 1932704 ; C-T (G to D) in $1307 / 01$ only \\
\hline $\mathrm{T} 10$ & 5 & 1932746-1933075 & 329 & Mb1750c & S & & & $7 \uparrow$ & $\begin{array}{l}\text { anti-toxin component of } \\
\text { toxin-antitoxin system }\end{array}$ & \\
\hline $\mathrm{T} 11$ & 6 & $2122959-2123263$ & 304 & Mb1914c & A & & $12 \uparrow$ & & $\begin{array}{l}\text { probable short-chain type } \\
\text { dehydrogenase/reductase }\end{array}$ & sSNP at $2122970 ;$ C-T in $2451 / 01$ only \\
\hline $\mathrm{T} 12$ & 2 & $2324463-2324571$ & 108 & Mb2110 & $\mathrm{S}$ & & & $3 \uparrow$ & $\begin{array}{l}\text { hypothetical protein } \\
\text { (frame-shifted in 2122/97) }\end{array}$ & \\
\hline $\mathrm{T} 13$ & 2 & $2328944-2329052$ & 108 & Mb2116c/pepE & S & & $2 \downarrow$ & $3 \downarrow$ & probable dipeptidase & \\
\hline T14 & 2 & 2329483-2329591 & 108 & Mb2117 & A & & $10 \downarrow$ & $10 \downarrow$ & $5^{\prime}-3^{\prime}$ exonuclease & $\begin{array}{l}\text { nSNP at 2329583; A-G (comp. strand; I to T) } \\
\text { in 2451/01 only }\end{array}$ \\
\hline $\mathrm{T} 15$ & 3 & 2868310-2868467 & 157 & Mb2607 & A & & $6 \downarrow$ & $8 \downarrow$ & hp (frame-shifted in 2122/97) & \\
\hline $\mathrm{T} 16$ & 2 & $2868506-2868614$ & 108 & Mb2607 & A & & $7 \downarrow$ & $7 \downarrow$ & hp (frame-shifted in 2122/97) & $\begin{array}{l}\text { nSNP at 2868616; A-G (stop to W) in } \\
\text { 2451/01 and 1307/01; }\end{array}$ \\
\hline $\mathrm{T} 17$ & 2 & $3075740-3075848$ & 108 & & । & & $5 \uparrow$ & $6 \uparrow$ & & \\
\hline T18 & 2 & 3075887-3075995 & 108 & & । & & $5 \uparrow$ & $6 \uparrow$ & & \\
\hline T19 & 2 & $3076622-3076730$ & 108 & & I & & $4 \uparrow$ & $4 \uparrow$ & & \\
\hline $\mathrm{T} 20$ & 2 & $3076769-3076877$ & 108 & & 1 & & $5 \uparrow$ & $5 \uparrow$ & & \\
\hline $\mathrm{T} 21$ & 2 & $3078728-3078836$ & 108 & & । & & $6 \uparrow$ & $7 \uparrow$ & & \\
\hline $\mathrm{T} 22$ & 2 & 3079169-3079277 & 108 & & । & & $4 \uparrow$ & $6 \uparrow$ & & \\
\hline $\mathrm{T} 23$ & 2 & 3079903-3080011 & 108 & & । & & $6 \uparrow$ & 7 & & \\
\hline T24 & 2 & $3844577-3844685$ & 108 & Mb3509c & $\mathrm{S}$ & & $3 \uparrow$ & $3 \uparrow$ & $\begin{array}{l}\text { possible acyltransferase } \\
\text { (frame-shifted in 2122/97) }\end{array}$ & \\
\hline $\mathrm{T} 25$ & 6 & $4155501-4155805$ & 304 & Mb3803/echA21 & A & & $5 \uparrow$ & & possible enoyl-coA hydratase & sSNP at 4155803; C-T (comp. strand) in 2451/01 only \\
\hline T26 & 2 & 4316987-4317095 & 108 & Mb3927c & A & & $3 \uparrow$ & & chp (frame-shifted in H37Rv) & \\
\hline
\end{tabular}


example, the amplicon array data had appeared to suggest that Mb1914c and echA21 were upregulated in 2451/01, but the oligo array data indicates that transcripts 11 and 25 , which are encoded within the co-ordinates encoded by those two genes, are actually encoded on the antisense strands. This apparent discrepancy can be rationalised once we consider that double stranded amplicon microarray probes cannot discriminate between transcripts encoded on the sense or antisense strands. Transcripts 11 and 25 can therefore be considered as potential antisense sRNAs (asRNA), which could be involved in translational or post-transcriptional control of the sense transcript. Other potential cis-encoded sRNAs detected using the arrays include T6, T14, and T15/T16 which are encoded on the antisense strands to Mb1618c, Mb2117 and Mb2607, respectively, and for each of these transcripts, their expression appears to be linked to the presence of a single SNP within the co-ordinates of the genes. The approximate boundaries of these transcripts can be derived from the genomic co-ordinates of the oligonucleotide probes that detect the expression of the transcript. Thus, the transcripts appear to be between 100-300 nt in size and the positions of the linked SNPs appear to be positioned either just upstream or within the predicted 5 'end of the transcripts (Figure 3). Three of the transcripts (T11, T14 and T25) are antisense to the central part of the sense encoded gene, while T6 is encoded antisense to the $5^{\prime}$ end of Mb1618c. As well as antisense transcripts, we also saw the differential expression of sense transcripts. The amplicon microarray data (confirmed by real time RT-PCR) indicated that nirB is strongly upregulated specifically in $1307 / 01$ in both in vitro and ex-vivo M $\phi$. An analysis of the oligonucleotide array data, however, indicates that there are two short transcripts, T1 and T2 (sense and antisense, respectively) that are encoded within the genomic co-ordinates of the nirB gene. T2 is the longer in size (255 vs. $155 \mathrm{nt}$ ) and more highly expressed (5 vs. 3 -fold) than T1, and both transcripts appear to be linked to the presence of a SNP that is located within the middle of T1 and approximately $50 \mathrm{nt}$ upstream of $\mathrm{T} 2$.

Some of the transcripts are bone fide gene sense strand mRNA transcripts, such as T9 and T10 which are encoded by Mb1749c and Mb1750c, respectively. Although it would appear that the two genes are transcribed separately, it is probable that the two transcripts are co-transcribed as the stop codon of Mb1750c overlaps the start codon of Mb1749c. Eight of the transcripts listed in Table 5 are encoded within intergenic regions, 7 of which are encoded within the polymorphic direct repeat (DR) locus. The DR locus of strains belonging to the M. tuberculosis complex has been suggested to constitute a CRISPR locus which have been shown in many species of bacteria to be involved in protection against exogenous foreign DNA such as plasmids and phage [25]. All the DR encoded transcripts are short (approx. $100 \mathrm{nt}$ ), straddle contiguous repeat and spacer sequences and show approximately 5 -fold higher levels of expression in 2451/01 and 1307/01 compared with $2122 / 97$ and 1121/01.

\section{Characterisation of differentially expressed cis asRNA}

The genomic co-ordinates of the oligonucleotide probes that detected the antisense species described above can only serve as approximate estimations as to their start and end points. Thus, we used 5' RLM-RACE (RNA Ligase Mediated Rapid Amplification of cDNA Ends) in an attempt to accurately define the transcriptional start sites (TSS) for the short sense transcript T2, and the antisense transcripts T6, T11 and T25 described in the above section (see Methods). These transcripts were chosen as their expression levels are high and their transcript lengths were considered to be sufficiently long to enable the RLM-RACE methodology to work. Table 6 details the sizes of the PCR products obtained after RLM-RACE was performed using oligonucleotide primers designed to sequences predicted for transcripts T6, T11 and T25. No PCR product was obtained for transcript T2. For each of the three transcripts, the TSS was determined to be a G residue, which is the most commonly used residue type for mycobacterial TSS's (Figure 4) [26]. For each of the T6, T11 and T25 transcripts, expression of the asRNAs was linked to the presence of a SNP ( $\mathrm{C}$ to $\mathrm{T}$ ) proximal to the 5 ' end of the asRNA. Strains exhibiting the ' $\mathrm{C}$ ' allele showed no expression of the asRNA, whilst the strain that showed expression had the ' $\mathrm{T}$ ' allele. An analysis of the nucleotide sequence in the vicinity of the SNPs reveals that for each of the three transcripts the SNP constitutes the 6th residue of a motif that has strong homology to the consensus sequence for the -10 element of Group A mycobacterial promoters (Figure 4) [26]. The finding that a ' $T$ ' residue is associated with expression is consistent with the consensus sequence which indicates that $86 \%$ of all -10 elements have a ' $T$ ' residue at the 6th residue position. Several residues that flank the -10 motif also show a degree of conservation. Sequence motifs which show strong homology to group A -35 elements are present $18-19$ bp upstream of the putative -10 elements, and the distances between the $-35,-10$ and TSS elements are consistent with those elements of the consensus sequence. No protein encoding open reading frames were detected within the $\mathrm{T} 6, \mathrm{~T} 11$ and T25 transcripts.

In a parallel study, high density oligonucleotide microarrays were also used to interrogate the transcriptomes of M. tuberculosis H37Rv, M. bovis BCG Pasteur, Mycobacterium caprae and $M$. bovis AN5 that had been grown in Middlebrook 7H9 media. As a result of these experiments, two asRNA species were found to be 


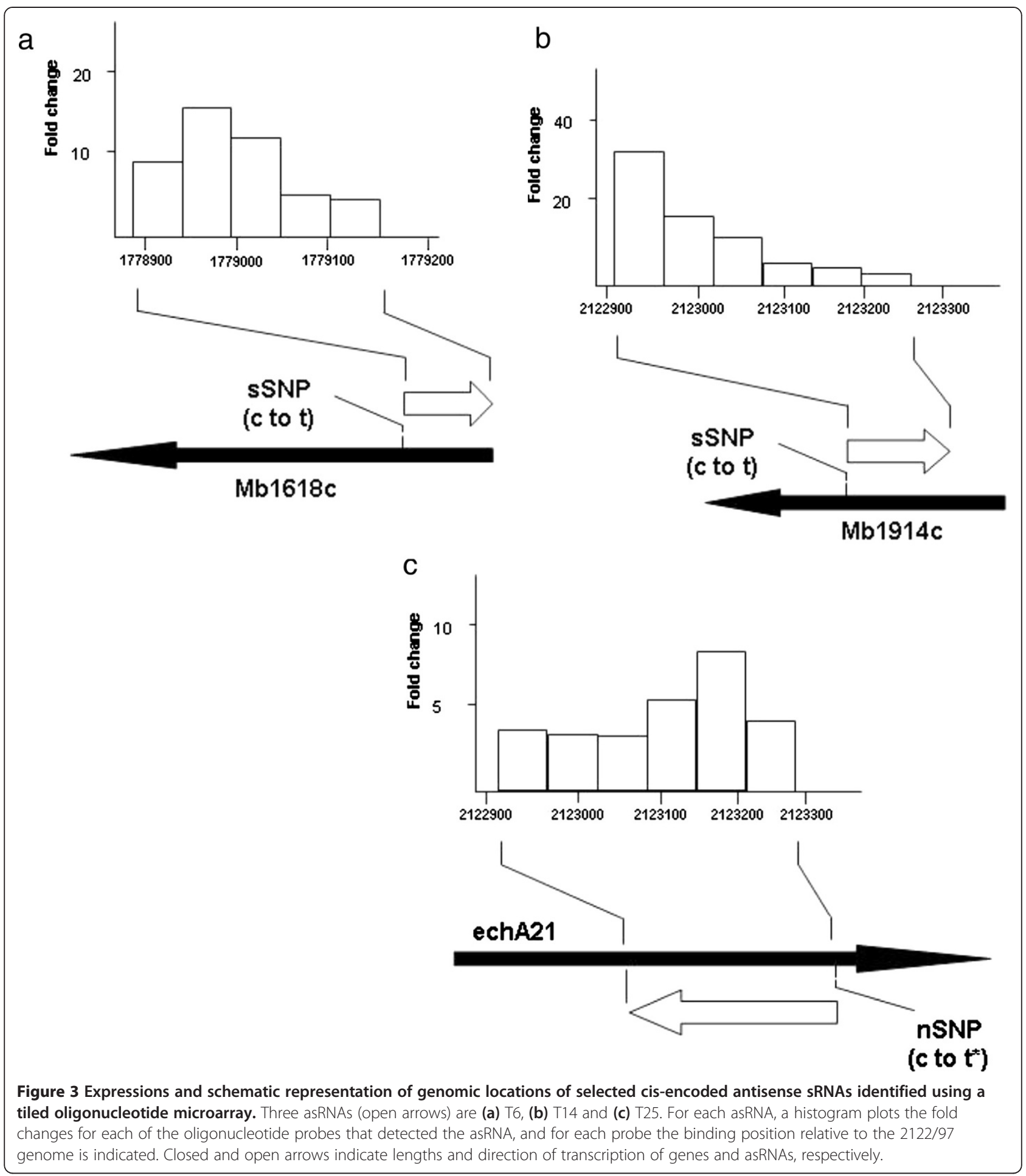

expressed within the antisense strands of the ino1 and narH genes of $M$. tuberculosis $\mathrm{H} 37 \mathrm{Rv}$, but not in any of the other 3 strains tested (data not shown). A comparison of nucleotide sequences of the orthologous genes across the species suggested that expressions of the as_sRNAs correlated with the presence of a SSNP (C to T transition at positions 50555 and 1292100 wrt $\mathrm{H} 37 \mathrm{Rv}$ genomic sequence for as_inol and as_narH, respectively) upstream of the asRNAs. Approximate information regarding the transcriptional start site was deduced from the binding co-ordinates of the probes that detected the transcripts. As with the M. bovis antisense sRNAs described 
Table 6 Determination of transcriptional start sites of antisense RNAs

\begin{tabular}{ccccc}
\hline Transcript & asRNA & $\begin{array}{c}\text { PCR } \\
\text { prod. size }\end{array}$ & $\begin{array}{c}\text { TSS } \\
\text { residue type }\end{array}$ & $\begin{array}{c}\text { TSS residue postn } \\
\text { (wrt 2122/97) }\end{array}$ \\
\hline T6 & mb1618c_as & 150 & $\mathrm{G}$ & 1778886 \\
T11 & mb1914c_as & 220 & $\mathrm{G}$ & 2122978 \\
T25 & echA21_as & 175 & $\mathrm{G}$ & 4155796 \\
\hline
\end{tabular}

above, the $\mathrm{T}$ residue associated with the expression of the $M$. tuberculosis asRNAs is part of a putative -10 element. A -35 element with an appropriate spacing to the -10 element was identified for as_ino1, but not for as_narH, suggesting that the as_narH promoter may belong to group $\mathrm{B}$ mycobacterial promoters that have a conserved -10 , but no -35 motif [26].

\section{Discussions}

The aim of this work was to define possible phenotypic variation across $M$. bovis field isolates through a combination of genome sequencing, comparative genomics and transcriptome analyses from both in vitro and ex vivo conditions. Using these approaches we uncovered a range of novel findings, the most striking of which was the realisation that genes that had been predicted to be differentially expressed based on amplicon-microarray data were in fact not upregulated, and that instead it was an antisense transcript that was showing differential expression. Analysing both transcriptome and genome sequence data allowed us to identify SNPs responsible for the transcription of antisense RNAs, with generation of a consensus -10 promoter sequence the likely mechanism. Our results suggest that data generated from amplicon arrays in the past may need to be revisited, as it is possible that some codingsequences identified as being differentially expressed were instead antisense transcripts.

With the growth of technologies such as high density tiled oligonucleotide microarrays and next generation sequencing there has been a rapid increase in the number of reports describing the existence of non-coding RNAs (ncRNAs) in bacteria. Non-coding RNAs broadly consist of two types, cis- and trans-encoded RNA. Trans RNA includes intergenic encoded RNA, while cis-encoded RNA includes $5^{\prime}$ and $3^{\prime}$ untranslated regions of mRNA and antisense RNA. To study the expressions of both cisand trans encoded ncRNA we used a high density oligonucleotide tiled microarray since our amplicon microarray was unable to detect intergenic transcripts or differentiate between sense and antisense transcripts. Previous studies using $M$. tuberculosis have identified substantial amounts of ncRNA encoded in both intergenic and intragenic regions $[7,8]$. We detected substantial amounts of ncRNAs

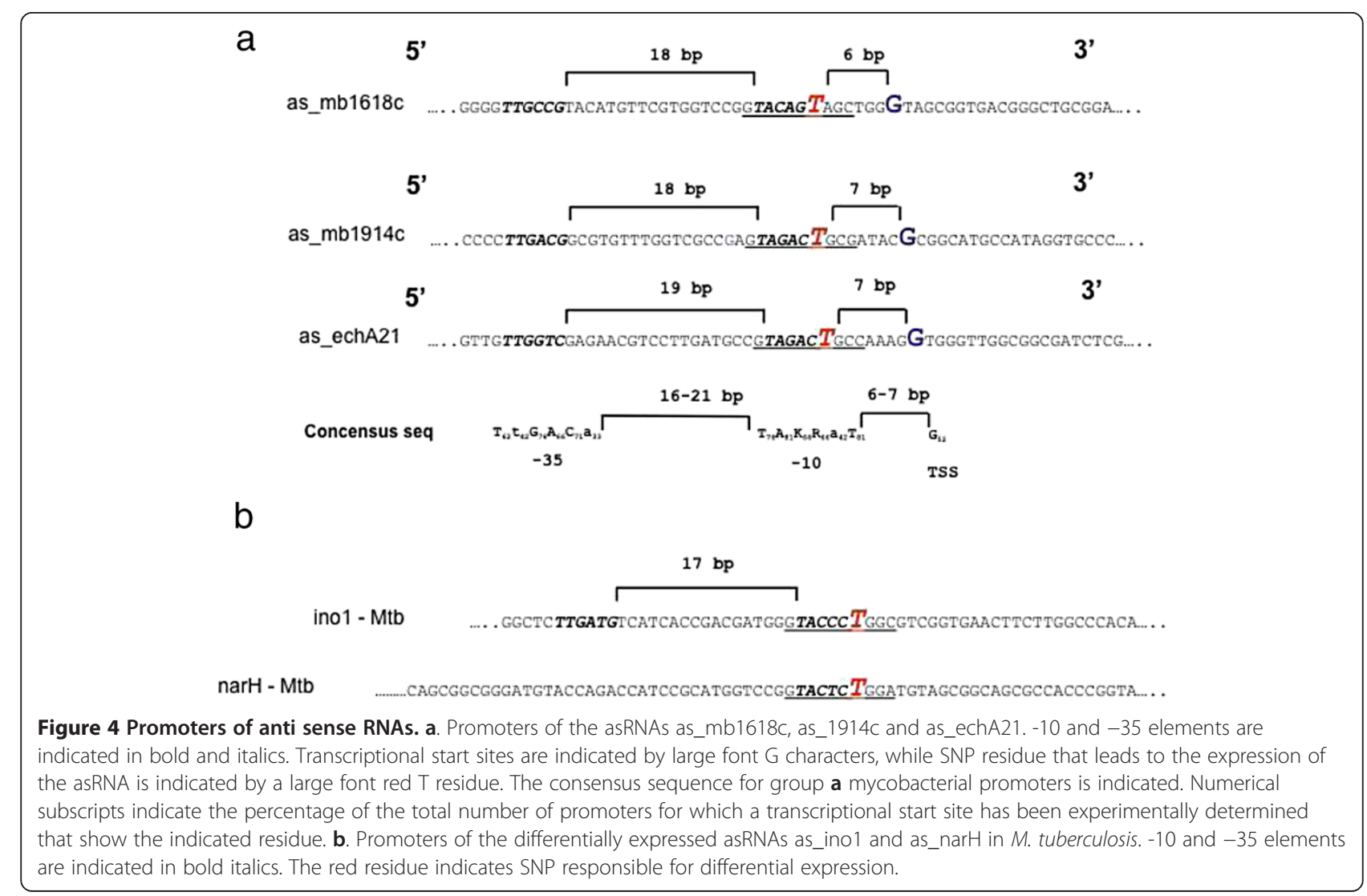


in $M$. bovis, including many instances of cis-antisense RNA species. Due to their perfect complementarity, cis asRNA form a duplex with the sense strand encoded transcript resulting in either degradation [27] or translation inhibition [28] of the sense mRNA. Antisense RNAs vary in length, ranging from 10 s to 1000 's of nucleotides and can be classified according to their encoded position with respect to the opposite sense encoded gene. Thus, they can be classified as $5^{\prime}$ or $3^{\prime}$ overlapping, while others are classified as internally located. The 1121/01 specific as_Mb1618c is an example of a $5^{\prime}$ overlapping asRNA, which is encoded antisense to gene Mb1618c which is predicted to express a secretory lipase. The location of the asRNA transcript suggests it may function to prevent translation of Mb1618c mRNA by steric hindrance of the ribosome binding site.

In the work presented here strain 2451/01 expressed two asRNAs, as_Mb1914c and as_echA21, that are not expressed by any of the other 3 strains. They are encoded within the central part of the opposite genes and are therefore likely to modulate the stability of the transcripts. Mb1914c encodes a short chain dehydrogenase while echA21 encodes an enoyl-CoA hydratase. Short chain dehydrogenases catalyse a wide range of functions so the precise function and identity of the substrate is difficult to deduce from sequence alone. Enoyl-CoA hydratases hydrate double carbon-carbon bonds of macromolecules and are vital in the metabolism of fatty acids. Both gene products would therefore appear to be involved in the metabolism of a macromolecule and their similar expression profiles in this strain could indicate involvement in the metabolism of the same molecule, or molecules that are of the same pathway.

In many instances, upregulation of asRNA negatively correlates with the transcription of the antisense gene [27], but in many cases expression of the antisense transcript has no effect on the transcription of the opposite gene. In our studies, expressions of as_Mb1618c, as_Mb1914c and as_echA21 did not appear to have any effect on the expressions of the opposite sense encoded genes (data not shown). We have shown that the expressions of the asRNAs are associated with the presence of SNPs, which are either synonymous or nonsynonymous with respect to the sense transcript, but upstream of the asRNA transcriptional start site. This highlights the fact that mutations can potentially affect expression of transcripts on both strands, and that the classification of a SNP is strand dependent. For each of the three asRNAs, the associated SNP was found to be located within a putative -10 promoter motif of group A mycobacterial promoters. The sixth residue of the -10 hexamer motif consensus sequence is a strongly conserved ' $\mathrm{T}$ ' residue, which is present in $81 \%$ of all group A mycobacterial promoter elements. Its importance is underlined by the finding that the strains that exhibit a ' $C$ ' residue at this position show no detectable expression of the asRNA, while strains having a ' $\mathrm{T}$ ' residue at this position exhibit expression.

Single nucleotide polymorphisms were found to be the most frequent form of genetic variation that exists between the isolates, with a total of 1013 SNPs detected across the three strains 1121/01, 2451/01 and 1307/01 compared to the reference strain $M$. bovis 2122/97. Non-synonymous SNPs, which include both non-sense and missense SNPs, are a class of SNPs most likely to impact on protein function and contribute to phenotypic variation. Non-sense SNPs, which results in the expression of a truncated polypeptide due to the introduction of a premature stop codon, were identified in five genes across the strains. Of these, we focussed our attentions on the non-sense SNP present in a gene encoding the LysR regulator, Mb2007c as mutations affecting regulators are likely to impact on the expression of one or more genes that are part of the regulon of the regulator and are therefore more likely to result in phenotypic variation. Experiments to compare the transcriptomes of a strain that exhibited the mutation with a strain overexpressing a functional regulator did not, however, reveal any differences. The reason for this unclear, but could reflect a requirement of the regulator for a co-inducer that was absent under the conditions of the experiment. The consequences of missense SNPs are more difficult to predict, as substitutions of one amino acid for another in a protein sequence do not necessarily lead to a change in protein function. However, for genes that are controlled by an autoregulatory mechanism, a mutation that affects the ability of the product of the gene to regulate itself will result in a change in expression of the gene. In our studies, we have shown that the presence of a missense mutation in a VapB type toxin encoding gene Mb1749c in strain 1307/01 results in the upregulation in expression of the toxin-antitoxin encoding pair of genes Mb1750c-Mb1749c due to the inability of the encoded proteins to self regulate themselves. Toxinantitoxin systems have a variety of proposed cellular functions including general regulation of mRNA stability levels in the cell [29]. Further experiments are required to fully understand the consequences of this mutation.

Genomic deletions have played an important role in the evolution of strains belonging to the mycobacterial complex [30], and in the derivation of the tuberculosis vaccine strain $M$. bovis BCG [31]. In addition to the previously described $6.8 \mathrm{~kb}$ gene deletion that is specific to strains having a spoligotype 17 pattern [6], we have identified a $1.6 \mathrm{~kb}$ multi-gene deletion that is specific to strain $1307 / 01$ and encompasses genes that are part of the DosR regulon. However, one of the deleted genes exists as a pseudo gene in DosR in strain 2122/97, so its importance to the biology of $M$. bovis is unlikely to be significant. 
Several other genes with internal deletions were detected but none of the encoded proteins have any significant similarity to any protein with a defined function.

\section{Conclusions}

In conclusion we have performed a comprehensive analysis of $4 M$. bovis strains of the most common molecular types circulating in GB. We show that while these strains show extensive similarities in their genetic make-up and gene expression profiles, they show distinct differences in the expression of a subset of genes. We provide functional data to show that SNPs can lead to the expression of antisense RNA, a finding with implications for how we define a 'silent' nucleotide change. Furthermore, we show that the interpretation of transcriptome data based solely on amplicon arrays could lead to artefacts due to expression of antisense transcripts, a caveat that needs to be kept in mind for previous studies of global expression analysis in bacteria.

\section{Methods}

\section{Bacterial strains, media and growth conditions}

For the $\mathrm{M} \phi$ infection experiments, bovine alveolar $\mathrm{M} \phi$ were cultivated in tissue culture media R10, which consisted of RPMI (Invitrogen) media plus $2 \mathrm{mM}$ glutamine, $10 \%$ calf fetal serum and 1\% amphotericin. Where used, antibiotics gentamycin and ampicillin were added at concentrations of 50 and $100 \mu \mathrm{g} / \mathrm{ml}$, respectively. $M$. bovis field strains were pre-grown in Middlebrook 7H9 broth supplemented with $10 \%$ albumindextrose catalase (ADC, Difco), 0.05\% Tween and $10 \mathrm{mM}$ pyruvate. Cultures were harvested in mid-logarithmic phase $\left(\mathrm{OD}_{600}\right.$ of $\left.0.3-0.8\right)$, washed and then resuspended in RPMI containing $0.05 \%$ Tween 80 .

\section{Isolation of bovine alveolar macrophages and infection with mycobacteria}

The lungs of a 6-8 week old male Holstein-Friesian calf were removed and a whole lung lavage procedure was performed to washout the alveolar $M \phi$. Briefly, 4-5 $\mathrm{x}$ $500 \mathrm{ml}$ aliquots of Hanks' Balanced Sterile Salts solution (HBSS) were used to infuse the lungs via the trachea, and the washings were pooled in a sterile beaker. The M $\phi$ cells contained in the washes were pelleted by centrifugation at $500 \mathrm{x} \mathrm{g}$ for $10 \mathrm{mins}$ at $4^{\circ} \mathrm{C}$, washed and then resuspended in R10 growth media supplemented with antibiotics (R10+) to a concentration of $1-2 \times 10^{7} / \mathrm{ml}$. Approximately $0.5-1.5 \times 10^{9} \mathrm{M} \phi$ were isolated per calf lung.

Vented $225 \mathrm{~cm}^{2}$ tissue culture flasks containing R10+ media were seeded with $3-4 \times 10^{7}$ alveolar $\mathrm{M \phi}$ and placed in a humidified $37^{\circ} \mathrm{C}$ incubator containing $5 \%$ $\mathrm{CO}_{2}$. Typically, 2-4 flasks were used per strain and time point. After 24 hrs, the growth media was decanted to remove non-adherent cells and then replaced with fresh R10+ media. After a further 24 hrs, the growth media was discarded and the M $\phi$ monolayer was washed with RPMI to remove traces of the antibiotic containing growth media. The monolayer was then covered with R10 media without antibiotics (R10-) and then infected with mid-logarithmic phase grown mycobacteria using an MOI of approximately 10:1 (bacilli: M $\phi$ ). The AlvM $\phi$ were incubated with mycobacteria for $4 \mathrm{hrs}$, after which the cell monolayers were washed with RPMI and then either processed for RNA extraction ( $4 \mathrm{hr}$ time point) or incubated in fresh $\mathrm{R} 10+$ media for a further $20 \mathrm{hrs}$ before being processed for RNA extraction (24 hr time point).

\section{Extraction and amplification of mycobacterial RNA from infected macrophages}

Mф cell monolayers were lysed using a guanidinium thiocyanate (GTC) containing solution. The lysed M $\phi$ 's were vortexed and passed twice through a $21 \mathrm{G}$ blunt ended needle to sheer host genomic DNA and thereby reduce the viscosity of the solution. Mycobacterial cells were then pelleted by centrifugation at $4600 \mathrm{rpm}$ for 20 mins at room temperature and washed with GTC solution to remove host genomic DNA. Cells were then resuspended in Trizol and RNA was extracted using the protocol outlined in Bacon et al. [32]. The amount of purified DNase-treated RNA recovered was of the order 100-500 ng per time point. RNA was amplified using the 'MessageAmp II-Bacteria RNA Amplification Kit' (Ambion) according to the manufacturers' instructions. Using an input of $100 \mathrm{ng}$ of unamplified RNA, 20-100 $\mu \mathrm{g}$ of amplified RNA was recovered.

\section{Amplicon microarray analysis}

For the in vitro growth experiments, three independent experiments (biological replicates) were carried out, and for each strain in each experiment two microarrays (technical replicates) were performed. Thus, for each strain 6 microarrays were performed. Three independent AlvM $\phi$ infection experiments were carried out and for each experiment two microarrays were performed for each of the control RPMI samples, and the 4- and 24 hrs postinfection samples. Cy5 and Cy3 fluorescently-labelled probes were synthesised from RNA and genomic DNA, respectively, and hybridised to whole genome M. bovis / M. tuberculosis microarrays. The array design is available in B $\mu \mathrm{G} @$ Sbase (accession number A-BUGS-31; http://bugs.sgul.ac.uk/A-BUGS-31) and also ArrayExpress (accession number A-BUGS-31). Details of probe synthesis, hybridization conditions and manufacture of the microarray can be found in Golby et al. 2008. Microarrays were scanned using an Affymetrix 428 Microarray scanner and scanned images were quantified using 
BlueFuse for Microarrays v3.2 software (BlueGnome). See Golby et al. 2008 for further details.

Normalisation was performed by dividing the log ratio of the Cy5 to Cy3 signal for every spot by the median of the log ratios for all spots, except control spots. A median absolute (MAD) scale transformation was applied to the normalised data from the pas an additional normalisation step. For every microarray, duplicate spots were averaged, and then the average expression of every gene across all technical replicate microarrays was calculated. Averages of the three biological replicates were used to compare gene expression between strains. For each gene, a moderated $\mathrm{t}$-test was applied and those genes with a P-value less than 0.05 were selected. From this gene list, those genes whose average expression differed by more than 2.5-fold between strains were selected. Fully annotated microarray data have been deposited in B $\mu \mathrm{G} @ S b a s e$ (accession number: E-BUGS-150; http://bugs.sgul.ac.uk/E-BUGS150 ) and also ArrayExpress (accession number: E-BUGS150).

\section{Oligonucleotide microarray analysis}

Experiments were performed in a similar way to that described for the in vitro amplicon array experiments, except that the RNA was purified using the mirVana miRNA Isolation kit (Ambion), which is designed to capture small (>20 nt) RNA species. RNA and genomic DNA were directly labelled with Cy5- and Cy3, respectively, using the ULS microRNA labelling kit (Kreatech), according to the manufacturer's instructions. Purified Cy5- and Cy3-labelled probes were co-purified and applied to an Agilent $40 \mathrm{~K}$ custom made tiled (10 nt overlap) 60-mer oligonucleotide microarray designed to the genomic sequence of $M$. bovis 2122/97/97. The array design is available in $\mathrm{B} \mu \mathrm{G} @$ Sbase (accession number A-BUGS-52; http://bugs.sgul.ac.uk/A-BUGS-52) and also ArrayExpress (accession number A-BUGS-52). Microarrays were hybridised at $65^{\circ} \mathrm{C}$ for $18 \mathrm{hrs}$ and then washed in Wash Buffer 1 (Agilent) at room temperature for 1 minute. The slides were then washed in Wash Buffer 2 (Agilent) at $37^{\circ} \mathrm{C}$ for 1 minute, dried and then scanned at $2 \mu \mathrm{m}$ using an Agilent DNA microarray scanner.

Tiling array data was analysed using the Limma package of R/Bioconductor [33]. The signal median was quantile normalised between arrays followed by a LOESS normalisation within arrays. Differential expression analysis was performed by pairwise comparison using linear models and empirical Bayes methods, and $\mathrm{P}$ values adjusted using the Benjamini and Hochberg's method to control for multiple testing. Fully annotated microarray data have been deposited in B $\mu \mathrm{G} @ S b a s e$ (accession number: E-BUGS-150; http://bugs.sgul.ac. uk/E-BUGS-150) and also ArrayExpress (accession number: E-BUGS-150).

\section{Whole genome sequencing of $\mathrm{M}$. bovis field isolates}

Whole genome paired end ( $2 \times 76 b p)$ sequencing was performed using Illumina HiSeq machines at the Wellcome Trust Sanger Institute (Hinxton, Cambridge). Raw sequence data was uploaded to the European Nucleotide Archive (ENA) and can be downloaded at http://www. ebi.ac.uk/ena/, accession numbers: ERX006616, ERX06617, ERX012284 and ERX012286. The FastQC (http://www. bioinformatics.babraham.ac.uk/projects/fastqc) program was used to analyse the quality of the raw data reads. Raw sequence data was trimmed to remove adapter sequences and nucleotides where the sequence quality score was below 20. Filtered reads were aligned to the $M$. bovis reference strain 2122/97 [10] with the SMALT alignment program (http://www.sanger.ac.uk/resources/software/smalt) using default settings. The average mean coverages for the sequenced strains 1121/01, 2451/01 and 1307/01 were 293, 224 and 234, respectively. Reads that did not map onto the reference genome were de novo assembled and blasted against the NCBI data base in order to find regions present in these strains but absent in 2122/01. Variant calling and the generation of consensus sequences were carried out using the SAMtools program suite (http://samtools.sourceforge.net). SNPs that had a minimum of quality score of 200 and had a minimum of four good quality forward (reverse) reads covering the SNP site and having the variant base were retained. The number of forward (reverse) reads mapping with good quality onto the SNP site having the same base as in the reference had to be less than $5 \%$ of the total number of forward (reverse) reads mapping with good quality onto the SNP site having the variant base. Sequences of 12 bases in length centred around SNP sites were blasted to the genome of the reference strain 2122/97, and any SNP within an area with a 90\% hit to another area of the genome were filtered out. This eliminated most of the SNPs within the repetitive PE-PGRS regions. For all sequenced strains, more than $99.9 \%$ of the genomes were covered by reads.

\section{Real time RT-PCR}

Quantitative real-time SYBR Green based PCR (qRT-PCR) experiments were performed using a RotorGene 3000 (Corbett research) as described by Golby et al. [23]. Fold changes were calculated using relative standard curve method and pcr controls included no template and no reverse transcriptase. Primer pair sequences are given in Additional file 5, available with the online version of this paper.

\section{Construction of the Rv1749c-Rv1750c and nirBD overexpressing plasmids}

The Rv1749c-Rv1750c overexpressing plasmids pPG106 and pPG107 were constructed by PCR amplification of a 907bp fragment encompassing $R v 1749 c-R v 1750 c-{ }^{-} R v 1751 c$ 
using primers tox_f and tox_r. For pPG106, the fragment was amplified using 2122/97/97 genomic DNA as a template, while pPG107 was amplified using 1307/01 gDNA. Both fragments were digested with SpeI and cloned into the SpeI cut mycobacterial attP-integrating shuttle vector pKINT (a gift from Douglas Young, Imperial College, London). Plasmids pPG108 and pPG109, which contain a $3.5 \mathrm{~kb}$ hsp'-nirB-nirD-'cobU fragment was constructed in several steps. Firstly, two $1.6 \mathrm{~kb}$ hsp'-nirB' PCR fragments were PCR amplified separately using primers nirB1_f and nirB1_r and 2122/97 and 1307/01 as genomic templates. Similarly, two $1.8 \mathrm{~kb}$ 'nirB-nirD-cobU fragments were amplified using primers nirB2_f and nirB2_r and genomic DNAs $2122 / 97$ and $1307 / 01$. The two PCR products were digested with SpeI and BamHI and then co-ligated into pKINT. Details concerning the nucleotide sequences of the pcr primer pairs are given in the Additional file 5.

\section{5'-RLM-RACE PCR}

Transcriptional start site mapping of was determined using the First Choice RNA ligase-mediated rapid amplification of cDNA ends (RLM-RACE) kit (Ambion) as per manufacturers instructions. Briefly, $10 \mathrm{ug}$ of total RNA was treated with calf intestinal phosphatase (CIP) and tobacco acid pyrophosphatase (TAP) before ligation of an RNA Adapter oligonucleotide to the $5^{\prime}$ ends of the mRNA transcripts. A random-primed reverse transcription reaction was carried out to generate cDNA and then nested PCR reactions were performed on the cDNA using combinations of adapter and gene specific primers. Details concerning the nucleotide sequences of the $5^{\prime}$ outer and inner adapter sequences as well as 3 ' outer and inner gene specific primers are given in the Additional file 5. PCR products generated using the $5^{\prime}$ inner adapter and 3 ' inner gene specific primers were sequenced by Sanger sequencing using the 3' inner gene specific primer.

\section{Animal ethics statement}

Animal work was carried out according to the UK Animal (Scientific Procedures) Act 1986. The study protocol was approved by the AHVLA Animal Use Ethics Committee (UK Home Office PCD number 70/6905).

\section{Availability of supporting data section}

Fully annotated microarray data have been deposited in B $\mu G @ S b a s e$ (accession number: E-BUGS-150; http:// bugs.sgul.ac.uk/E-BUGS-150) and also ArrayExpress (accession number: E-BUGS-150). Raw sequence data was uploaded to the European Nucleotide Archive (ENA) and can be downloaded at http://www.ebi.ac.uk/ena/data/view/ ERX006616-ERX06617,ERX012284-ERX012286.

\section{Additional files}

\begin{abstract}
Additional file 1: SNPs identified across sequenced strains. Additional file 2: Large deletions identified by NGS in sequenced strains.
\end{abstract}

Additional file 3: Fold change differences in gene expression in M. bovis field isolates 1121, 2451 and 1307 compared to 2122 .

Cells shaded in red indicate upregulation, green indicate downregulation and empty cells indicate no change in expression.

Additional file 4: Differential expression of RNA transcripts as detected by a tiled oligonucleotide microarray. Up and down arrows indicate fold up- and downregulation, respectively, and empty cells indicate no change in expression. *Strand, A indicates antisense, S, sense and I, intergenic.

Additional file 5: Details of oligonucleotides used in PCR, RT-PCR and RLM-RACE experiments.

\section{Competing interests}

The authors declare that they have no competing interests.

\section{Authors' contributions}

PG carried out the culturing of organisms, macrophage infections, microarrays, qRT-PCR, cloning, analysis of sequence data and writing of the manuscript. JN performed analysis of the Illumina sequence and PCR amplicon microarray data. AW and $\mathrm{JH}$ performed the analysis of the Agilent oligonucleotide microarray data and assisted in the uploading of the microarray data into the BHG@Sbase database. MAQ and SB were responsible for the Illumina sequencing. SH contributed to the analysis of the Illumina sequence data. NS contributed to the writing and critical review of the manuscript. RGH was a co-investigator who helped to guide the project. SG was the principle investigator on the project who guided the research and helped in the writing of the manuscript. All authors read and approved the final manuscript.

\section{Acknowledgements}

We would like to acknowledge Kate Gould for her help in carrying out the oligonucleotide microarrays and Denise Waldron for her assistance in uploading of the microarray data into BuG@Sbase. We also thank Mark Chambers for comments on the manuscript, and BBSRC and Defra for funding the work (Grant no. BB/E018491/1).

\section{Author details}

${ }^{1}$ Animal Health and Veterinary Laboratories Agency, Woodham Lane, New Haw Addlestone, Surrey KT15 3NB, UK. ²Division of Clinical Sciences, Bacterial Microarray Group, Centre for Infection \& Immunity, St George's, University of London, Cranmer Terrace, London SW17 ORE, UK. ${ }^{3}$ The Wellcome Trust Sanger Institute, Hinxton, Cambridgeshire, UK. ${ }^{4}$ UCD School of Veterinary Medicine and UCD Conway Institute, University College Dublin, Dublin 4, Ireland.

Received: 4 June 2013 Accepted: 26 September 2013

Published: 17 October 2013

\section{References}

1. Monaghan ML, Doherty ML, Collins JD, Kazda JF, Quinn PJ: The tuberculin test. Vet Microbio/ 1994, 40(1-2):111-124.

2. Kamerbeek J, Schouls L, Kolk A, van Agterveld M, van Soolingen D, Kuijper S, Bunschoten A, Molhuizen H, Shaw R, Goyal M, et al: Simultaneous detection and strain differentiation of Mycobacterium tuberculosis for diagnosis and epidemiology. J Clin Microbio/ 1997, 35(4):907-914

3. Frothingham $\mathrm{R}$, Meeker-O'Connell WA: Genetic diversity in the Mycobacterium tuberculosis complex based on variable numbers of tandem DNA repeats. Microbiology 1998, 144(Pt 5):1189-1196.

4. Smith NH, Dale J, Inwald J, Palmer S, Gordon SV, Hewinson RG, Smith JM: The population structure of Mycobacterium bovis in Great Britain: clonal expansion. Proc Natl Acad Sci USA 2003, 100(25):15271-15275.

5. Winder CL, Gordon SV, Dale J, Hewinson RG, Goodacre R: Metabolic fingerprints of Mycobacterium bovis cluster with molecular type: 
implications for genotype-phenotype links. Microbiology 2006, 152(Pt 9):2757-2765.

6. Wheeler PR, Brosch R, Coldham NG, Inwald JK, Hewinson RG, Gordon SV: Functional analysis of a clonal deletion in an epidemic strain of Mycobacterium bovis reveals a role in lipid metabolism. Microbiology 2008, 154(Pt 12):3731-3742.

7. Arnvig KB, Young DB: Identification of small RNAs in Mycobacterium tuberculosis. Mol Microbiol 2009, 73(3):397-408.

8. Arnvig KB, Comas I, Thomson NR, Houghton J, Boshoff HI, Croucher NJ, Rose G, Perkins TT, Parkhill J, Dougan G, et al: Sequence-based analysis uncovers an abundance of non-coding RNA in the total transcriptome of Mycobacterium tuberculosis. PLoS Pathog 2011, 7(11):e1002342.

9. Smith NH, Upton P: Naming spoligotype patterns for the RD9-deleted lineage of the Mycobacterium tuberculosis complex; www. Mbovis.org Infect Genet Evol 2012, 12(4):873-876.

10. Garnier T, Eiglmeier K, Camus JC, Medina N, Mansoor H, Pryor M, Duthoy S, Grondin S, Lacroix C, Monsempe C, et al: The complete genome sequence of Mycobacterium bovis. Proc Natl Acad Sci USA 2003, 100(13):7877-7882.

11. Albers CA, Lunter G, MacArthur DG, McVean G, Ouwehand WH, Durbin R: Dindel: accurate indel calls from short-read data. Genome Res 2011, 21(6):961-973

12. Mostowy S, Inwald J, Gordon S, Martin C, Warren R, Kremer K, Cousins D, Behr MA: Revisiting the evolution of Mycobacterium bovis. J Bacteriol 2005, 187(18):6386-6395.

13. Boon C, Dick T: Mycobacterium bovis BCG response regulator essential for hypoxic dormancy. J Bacteriol 2002, 184(24):6760-6767.

14. Van Gelder RN, von Zastrow ME, Yool A, Dement WC, Barchas JD, Eberwine $J \mathrm{H}$ : Amplified RNA synthesized from limited quantities of heterogeneous cDNA. Proc Natl Acad Sci USA 1990, 87(5):1663-1667.

15. Pandey DP, Gerdes K: Toxin-antitoxin loci are highly abundant in freeliving but lost from host-associated prokaryotes. Nucleic Acids Res 2005, 33(3):966-976

16. Arcus VL, McKenzie JL, Robson J, Cook GM: The PIN-domain ribonucleases and the prokaryotic VapBC toxin-antitoxin array. Protein Eng Des Sel 2011, 24(1-2):33-40.

17. Bukowski M, Rojowska A, Wladyka B: Prokaryotic toxin-antitoxin systemsthe role in bacterial physiology and application in molecular biology. Acta Biochim Pol 2011, 58(1):1-9.

18. Maddocks SE, Oyston PC: Structure and function of the LysR-type transcriptional regulator (LTRR) family proteins. Microbiology 2008, 154(Pt 12):3609-3623.

19. Moreno-Vivian C, Cabello P, Martinez-Luque M, Blasco R, Castillo F: Prokaryotic nitrate reduction: molecular properties and functional distinction among bacterial nitrate reductases. J Bacteriol 1999, 181 (21):6573-6584.

20. Tiffert $Y$, Supra P, Wurm R, Wohlleben W, Wagner R, Reuther J: The Streptomyces coelicolor GlnR regulon: identification of new $G \ln R$ targets and evidence for a central role of $\mathrm{G} \ln R$ in nitrogen metabolism in actinomycetes. Mol Microbiol 2008, 67(4):861-880.

21. Duan J, Wainwright MS, Comeron JM, Saitou N, Sanders AR, Gelernter J, Gejman PV: Synonymous mutations in the human dopamine receptor D2 (DRD2) affect mRNA stability and synthesis of the receptor. Hum Mol Genet 2003, 12(3):205-216.

22. Capon F, Allen MH, Ameen M, Burden AD, Tillman D, Barker JN, Trembath RC: A synonymous SNP of the corneodesmosin gene leads to increased mRNA stability and demonstrates association with psoriasis across diverse ethnic groups. Hum Mol Genet 2004, 13(20):2361-2368.

23. Golby P, Hatch KA, Bacon J, Cooney R, Riley P, Allnutt J, Hinds J, Nunez J, Marsh PD, Hewinson RG, et al: Comparative transcriptomics reveals key gene expression differences between the human and bovine pathogens of the Mycobacterium tuberculosis complex. Microbiology 2007, 153(Pt 10):3323-3336.

24. Bitter W, Houben EN, Bottai D, Brodin P, Brown EJ, Cox JS, Derbyshire K, Fortune SM, Gao LY, Liu J, et al: Systematic genetic nomenclature for type VII secretion systems. PLoS Pathog 2009, 5(10):e1000507.

25. Sorek R, Kunin V, Hugenholtz P: CRISPR-a widespread system that provides acquired resistance against phages in bacteria and archaea. Nat Rev Microbiol 2008, 6(3):181-186.

26. Gomez M, Smith I: Determinants of Mycobacterial Gene Expression. In Molecular Genetics of Mycobacteria. Washington, D.C: ASM Press; 2000:111-129.
27. Duhring U, Axmann IM, Hess WR, Wilde A: An internal antisense RNA regulates expression of the photosynthesis gene isiA. Proc Natl Acad Sci USA 2006, 103(18):7054-7058.

28. Kawano M, Aravind L, Storz G: An antisense RNA controls synthesis of an SOS-induced toxin evolved from an antitoxin. Mol Microbiol 2007, 64(3):738-754.

29. Thomason MK, Storz G: Bacterial antisense RNAs: how many are there, and what are they doing? Annu Rev Genet 2010, 44:167-188.

30. Brosch R, Pym AS, Gordon SV, Cole ST: The evolution of mycobacterial pathogenicity: clues from comparative genomics. Trends Microbiol 2001, 9(9):452-458.

31. Calmette A, Guerin C: Sur quelques proprietes du bacille tuberculeux d'origine, cultive sur la bile de boeuf glycerinee. C R Acad Sci Paris 1909, 149:716-718.

32. Bacon J, James BW, Wernisch L, Williams A, Morley KA, Hatch GJ, Mangan $J A$, Hinds J, Stoker NG, Butcher PD, et al: The influence of reduced oxygen availability on pathogenicity and gene expression in Mycobacterium tuberculosis. Tuberculosis (Edinb) 2004, 84(3-4):205-217.

33. Smyth GK: Linear models and empirical bayes methods for assessing differential expression in microarray experiments. Stat Appl Genet Mol Biol 2004, 3:3.

doi:10.1186/1471-2164-14-710

Cite this article as: Golby et al:: Genome-level analyses of

Mycobacterium bovis lineages reveal the role of SNPs and antisense transcription in differential gene expression. BMC Genomics 2013 14:710.

\section{Submit your next manuscript to BioMed Central and take full advantage of:}

- Convenient online submission

- Thorough peer review

- No space constraints or color figure charges

- Immediate publication on acceptance

- Inclusion in PubMed, CAS, Scopus and Google Scholar

- Research which is freely available for redistribution 